\title{
Real-Space and Energy Representations for the Interface Roughness Scattering in Quantum-Well Structures
}

\author{
S. K. Lyo \\ Sandia National Laboratories, Albuquerque, N. M. 87185
}

We show that the real space representation of the interface-roughness as a fluctuating potential in the coordinate space is equivalent to the usual energy- fluctuation representation for intrasublevel scattering in a single quantum well with a generally shaped confinement-potential profile. The coordinate picture is, however, more general and can be used for higher-order effects and multi-sublevel scattering in coupled multi-quantum-well structures.

PACS: 72.10.Fk, 72.80.Ey, 73.61.Ey, 73.61.Ga

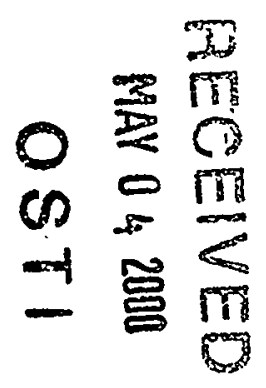




\section{DISCLAIMER}

This report was prepared as an account of work sponsored by an agency of the United States Government. Neither the United States Government nor any agency thereof, nor any of their employees, make any warranty, express or implied, or assumes any legal liability or responsibility for the accuracy, completeness, or usefulness of any information, apparatus, product, or process disclosed, or represents that its use would not infringe privately owned rights. Reference herein to any specific commercial product, process, or service by trade name, trademark, manufacturer, or otherwise does not necessarily constitute or imply its endorsement, recommendation, or favoring by the United States Government or any agency thereof. The views and opinions of authors expressed herein do not necessarily state or reflect those of the United States Government or any agency thereof. 


\section{DISCLAIMER}

Portions of this document may be illegible in electronic image products. Images are produced from the best available original document. 
Interface roughness is present in all artificially fabricated layered structures. It causes unavoidable linewidths in optical and transport spectroscopy and also limits the low-temperature mobility in modulation-doped semiconductor quantum wells (QW's). Sasaki et al. demonstrated experimentally and theoretically that interface-roughness scattering is a dominant scattering mechanism for the low-temperature mobility in modulation-doped narrow QW's. [1] In this treatment, the energy fluctuation due to the interface roughness for intrasublevel scattering is given by

$$
\delta E\left(\mathbf{r}_{\|}\right)=\frac{\partial E}{\partial L} \delta L\left(\mathbf{r}_{\|}\right),
$$

where $E$ is the sublevel energy and $\delta L\left(\mathbf{r}_{\|}\right)$is the fluctuation of the well width $L$ at the in-plane position vector $\mathbf{r}_{\|}$. The scattering potential $\delta E\left(\mathbf{r}_{\|}\right)$is thus independent of the coordinate $z$ in the growth direction and is suitable only for lowest-order intrasublevel scattering. The advantage of this picture is that theoretical results calculated from Eq. (1) have no explicit dependence on the confinement wave function $\psi(z)$. At the same time, however, the fluctuation energy $\delta E\left(\mathbf{r}_{\|}\right)$in Eq. (1) is inadequate for treating more complicated problems such as higher-order scattering effects or intersublevel scattering in single and coupled multi-QW's. It is also inadequate for treating intrasublevel scattering in coupled double- or multi-QW's with multiple interfaces. In this paper, we study a more general form for the scattering potential which reduces to Eq. (1) for a single-sublevel problem in a single QW.

A natural microscopic picture for the fluctuation energy is to write

$$
\delta E\left(\mathbf{r}_{\|}, z\right)= \pm V_{ \pm} \delta L\left(\mathbf{r}_{\|}\right) \delta\left(z \pm \frac{1}{2} L\right),
$$

where $\delta(z)$ is the Dirac delta function and $V_{l}=V_{+}\left(V_{r}=V_{-}\right)$is the potential-energy discontinuity at the left (right) interface at $z=-L / 2(z=L / 2)$ as shown in Fig. 1. Here $\delta L\left(\mathbf{r}_{\|}\right)$can take different values at the right and left interfaces, although we do not introduce separate 
symbols for simplicity. While the expression in Eq. (2) is intuitively deduced from a standard perturbation theory, its validity and equivalence to the expression in Eq. (1) should be examined for a general confinement-potential structure, including the often used limit of infinitely deep QW's (i.e., band offsets) with $V_{ \pm} \rightarrow \infty$. The effect of the potential in Eq. (2) on the distortion of the confinement wave functions can be included by going beyond the Born approximation. [2]

The inter- and intrasublevel matrix element of the potential in Eq. (2) is given by

$$
\left\langle f\left|\delta E\left(\mathbf{r}_{\|}, z\right)\right| i\right\rangle= \pm V \delta L\left(\mathbf{r}_{\|}\right) \psi_{f}^{*}(\mp L / 2) \psi_{i}(\mp L / 2),
$$

where $\psi_{i}(z)$ and $\psi_{f}(z)$ are sublevel functions. In order to show that Eq. (3) reduces to Eq. (1) for intrasublevel scattering for a general confinement-potential structure in a single QW, we prove the following identity

$$
\left\langle\psi\left|\delta E\left(\mathbf{r}_{\|}, z\right)\right| \psi\right\rangle=-V_{-} \delta L\left(\mathbf{r}_{\|}\right)|\psi(L / 2)|^{2}=\frac{\partial E}{\partial L} \delta L\left(\mathbf{r}_{\|}\right),
$$

where $\psi(z)$ is any sublevel function with an eigenvalue $E$ defined below in Eq. (5). Equation (4) is written only for the layer fluctuation at the right interface for simplicity.

For this purpose, we write the Schroedinger equations as

$$
\begin{aligned}
& H \psi=E \psi, \\
& H=H_{o}+V(z), \\
& V(z)=V_{l} \theta\left(z_{l}-z\right)+V_{r} \theta\left(z-z_{r}\right),
\end{aligned}
$$

where $H$ is the Hamiltonian without interface fluctuations and $V(z)$ represents the discontinuous potential-energy steps at the left $\left(V_{l}=V_{+}\right)$and right $\left(V_{r}=V_{-}\right)$interfaces at $z=z_{l}, z_{r}$ (Fig.1). In Eq. (5b), $H_{\mathrm{o}}$ is the rest of the Hamiltonian including an arbitray band-bending potential-profile around the interfaces in the presence of ionized dopants. We assume that there is no correlation between the interface fluctuations at the right and left interfaces at 
any position $\mathbf{r}_{\|}$and consider only the fluctuation $\delta z_{r}$ at the right interface for convenience. By taking a derivative with respect to $z_{r}$ on both sides of Eq. (5a), using $\partial V(z) / \partial z_{r}=-V_{r} \delta(z$ $\left.-z_{r}\right)$, and taking an inner product from the left side with $\psi^{*}$, we find

$$
\frac{\partial E}{\partial z_{r}}=-V_{r}\left|\psi\left(z_{r}\right)\right|^{2} .
$$

This expression is identical to Eq. (4) if one identifies $\delta z_{r}=\delta L\left(\mathbf{r}_{\|}\right)$. Note that the effect of the wave-function distortion due to $\delta z_{r}$ (i.e., $\partial \psi(z) / \partial z_{r} \neq 0$ ) is included in the above derivation.

The interesting general relationship in Eq. (6) can be verified for the eigenvalues and eigenfunctions of a square-well potential, for example, by tediously calculating the eigenvalues and the eigenfunctions. One can also show that the relationship holds even for an infinitely deep well. In this limit (i.e., $V_{r} \rightarrow \infty$ ), the wave function $\psi\left(z_{r}\right)$ in Eq. (6) becomes infinitesimally small (i.e., $\left|\psi\left(z_{r}\right)\right| \sim 1 /\left|V_{r}\right|^{1 / 2} \rightarrow 0$ ) at the interface, cancelling the factor $V_{r}$

yielding $\partial E / \partial z_{r}=-\hbar^{2} /\left(m^{*} L^{3}\right)$ for the ground sublevel as expected.

In summary, we have shown that the coordinate representation of the interface-roughness scattering potential in Eq. (2) reduces to the usual energy-fluctuation model in Eq. (1) for the simple case of intrasublevel scattering in a single quantum well. The representation in Eq. (2) allows for different types of layer fluctuations on the interfaces in contrast to that in Eq. (1) and is useful in treating multi-sublevel scattering in coupled multi-QW's. [3]

\section{ACKNOWLEDGMENTS}

Sandia is a multiprogram laboratory operated by Sandia corporation, a Lockheed Martin Company, for the U.S. DOE under Contract No.DE-AC04-94AL85000. 


\section{References}

1. H. Sasaki, T. Noda, K. Hirakawa, M. Tanaka, and T. Matsusue, Appl. Phys. Lett. 51, 1934 (1987).

2. T. Ando, A. B. Fowler, and F. Stern, Rev. Mod. Phys. 54, 437 (1982).

3. D. Huang and S. K. Lyo, J. Phys. [Condens. Matter], in press (2000). 


\section{Figure Captions}

Fig. 1 Confinement potential-energy profile for the electrons with an arbitrary band bending. The quantities $V_{l}$ and $V_{r}$ represent potential-energy discontinuities at the left and right interfaces at $z=z_{l}$ and $z=z_{r}$ 
$\frac{7}{9}$

Confinement-potential Energy

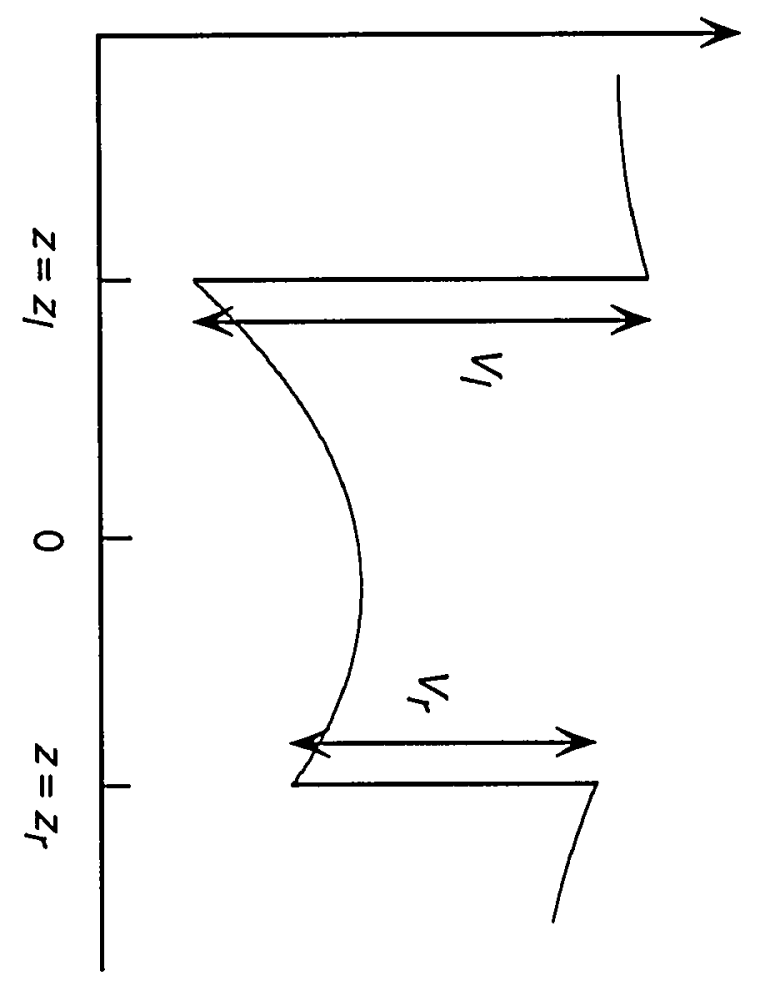

